\title{
INFERENCES FROM OPTICAL PROPERTIES CONCERNING THE SURFACE TEXTURE AND COMPOSITION OF ASTEROIDS
}

\author{
BRUCE HAPKE \\ University of Pittsburgh
}

The optical properties of the asteroids are compared with those of the Moon and various terrestrial, lunar, and meteoritic materials. It is concluded that the surfaces of most of the asteroids are covered with at least a thin layer of unconsolidated, fine-grained powder similar to lunar soil. None of the asteroids appear to have compositions corresponding to pure nickel/iron meteorites.

The picture that most of us have in our minds of a typical asteroid is probably of a large, irregularly shaped chunk of iron, unrusted by exposure to oxygen or water, and with a surface kept clean and dust free by the sandblasting effect of repeated micrometeorite impacts. However, consideration of the known optical properties of asteroids suggests a rather different model. It is the purpose of this paper to review briefly the optical characteristics of asteroids and to compare them with other extraterrestrial and terrestrial materials to obtain information concerning the nature of the outer surfaces of the minor planets.

\section{OPTICAL PROPERTIES OF ASTEROIDS}

The optical characteristics of the asteroids that this paper will be concerned with include the visual albedo, $U B V$ color indexes, brightness-phase curve, and polarization-phase curve. These properties are summarized in table $I$ and figure 1. In the tables and figures, the following were used: the visual geometric albedo; $U-B$ and $B-V$, differences between $U B V$ color indexes relative to the Sun; $\partial m_{\nu} / \partial \alpha$, the slope of the apparent visual magnitude $m_{v}$ versus phase angle $\alpha$ for $5^{\circ}<\alpha<25^{\circ}$; the opposition effect defined as the ratio of brightness at $\alpha=1^{\circ}$ to that at $\alpha=5^{\circ}$; the value of polarization at the minimum of the polarization-phase curve; $\alpha^{-}$, phase angle at which the minimum occurs; $\alpha_{0}$, phase angle (other than $0^{\circ}$ and $180^{\circ}$ ) at which the polarization is zero. The data for table I and figure 1 are taken primarily from the review paper by Gehrels (1970) and from these additional sources: Dollfus (1961), Harris (1961), Miner and Young (1969), Gehrels (1956), Allen (1963), 


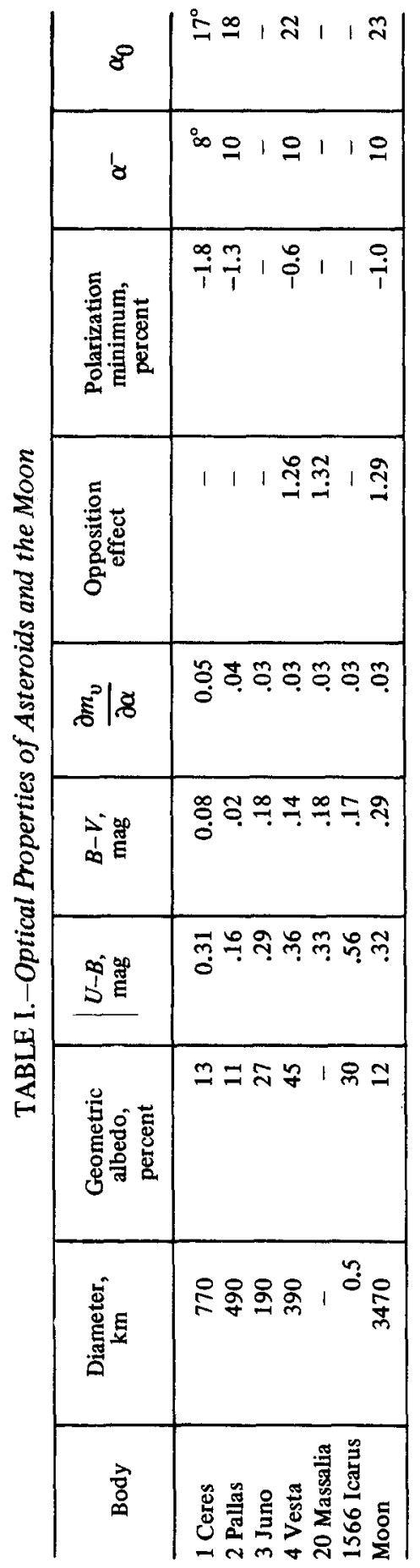




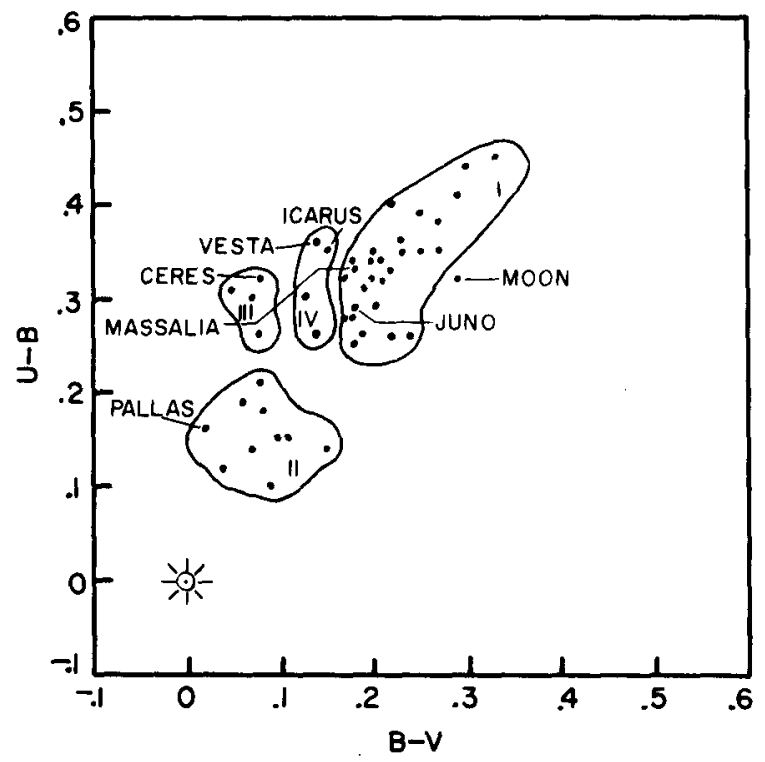

Figure 1.-Differences between $U B V$ indexes of asteroids and the Moon relative to the Sun. Also see figure 1 of the paper by Chapman, Johnson, and MoCord. 1

Goldstein (1968), and Veverka (in this volume ${ }^{2}$ ). Included in table I and figure 1 are data for the Moọn for comparison.

Because of the faintness of the asteroids, detailed optical data exist only for relatively few bodies. To calculate the geometric albedo from the absolute magnitude, the diameter must be known; but only a few of the largest of the minor planets show disks. (See the paper by Dollfus. ${ }^{3}$ ) Detailed reflectivity curves have been published for only one asteroid, Vesta (McCord, Adams, and Johnson, 1970); for the rest, only the $U B V$ indexes are known. ${ }^{4}$ The degree to which the optical properties of the larger bodies are representative of all asteroids is not clear. Also, among the fainter asteroids, high-albedo objects, such as Vesta, will be preferentially discovered and observed over low-albedo bodies, such as Pallas.

\section{OPTICAL PROPERTIES OF GEOLOGICAL MATERIALS}

Because the only optical information available for most of the asteroids are their $U B V$ indexes, the greatest attention will be given to the interpretation of these spectral data. To facilitate comparison with the various laboratory

\footnotetext{
${ }^{1}$ See p. 55.

${ }^{2}$ See pp. 79 and 91.

${ }^{3}$ See pp. 25, 30, and $\mathrm{xv}$.

${ }^{4}$ Editorial note: Additional data now are available in the paper by Chapman, Johnson, and McCord, p. 51.
} 
materials, it is necessary to discuss the processes that influence the reflection of light from complex surfaces in the near-UV, visible, and near-IR wavelength region.

\section{Absorption Processes}

Materials of geological interest absorb light by a variety of processes, which will be described briefly. For further information, references such as Garbuny (1965) and Burns (1970) should be consulted.

Metallic Conductivity.-Metals contain electrons that are not bound to any particular atom but are free to move about the lattice. The electrons are able to respond rapidly to varying electromagnetic fields and rearrange themselves to prevent the penetration of fields into the interior of the metal. The coefficient for the absorption of electromagnetic waves is thus extremely high in the UV-IR range.

Charge Exchange. - A number of nonmetals contain cations that can exist in more than one valence state. If these cations are not separated by too great a distance in the solid-state lattice, then certain electrons, although momentarily bound to a given ion, may nevertheless be able to move about the lattice from one atom to another by a series of oxidation-reduction reactions. The absorption coefficients of these materials are also very high. Important examples are magnetite $\mathrm{Fe}_{3} \mathrm{O}_{4}$ and ilmenite $\mathrm{FeTiO}_{3}$.

Electronic Transitions. - Several transition elements have $d$ electron shells that are degenerate in the free ion. However, when the ion is in a solid-state lattice, the anisotropy of the electric fields from the surrounding anions removes the degeneracy and may produce states separated by energies corresponding to UV-IR wavelengths. The most important element involved in this type of absorption is iron because of its cosmological abundance. Other elements that also may be significant in determining the colors of certain minerals and glasses are $\mathrm{Ti}, \mathrm{Cr}$, and $\mathrm{Mn}$. The ferrous ion $\mathrm{Fe}^{2+}$ has a weak electron-transition band near $1000 \mathrm{~nm}$. This band has been effectively exploited by McCord and his coworkers (McCord and Johnson, 1970; McCord, Adams, and Johnson, 1970; see the paper by Chapman in this volume ${ }^{5}$ ) for the remote identification of lunar and asteroidal materials. The ferrous band is especially useful because its exact position depends on the detailed mineralogy of the crystals in which the ion is located and thus often allows identification of the type of rock present on the surface of a body. The ferric ion $\mathrm{Fe}^{3+}$ has an extremely strong absorption band near $235 \mathrm{~nm}$; this band has not yet been exploited astrophysically because of the ozone cutoff in Earth's atmosphere. Paradoxically, the presence of iron ions can cause a mineral to be red, green, or blue, depending on the valence states of the iron.

Other.-Other processes, such as band-gap conductivity and color centers, are not discussed here either because they are not important for materials of

\footnotetext{
${ }^{5}$ See p. 51 .
} 
interest to this paper or because their region of light absorption lies outside the range of UV.IR wavelengths.

\section{Scattering Processes}

Light diffusely reflected from a complex surface consists of rays that have been scattered by two processes: (1) rays that have been reflected from the outer surfaces of grains and (2) rays that have penetrated several wavelengths into the interior of grains and subsequently have been scattered out of the surface by some irregularity. The reflection coefficient for surface-scattered rays is determined, according to the well-known Fresnel laws, by the index of refraction and absorption coefficient of a grain of surface material. The intensity of the volume-scattered ray is determined primarily by the absorption coefficient. For a weakly absorbing material, such as $\mathrm{MgO}$ or pure enstatite $\mathrm{MgSiO}_{3}$, the reflection is dominated by volume scattering and the albedo of the substance is high. For a strongly absorbing material, such as a metal, the reflection is almost entirely by surface scattering; the albedo is low because each reflection is a rather inefficient process. A high albedo almost invariably implies a small volume-absorption coefficient.

\section{UBV Colors}

The $U B V$ properties of a number of terrestrial, lunar, and meteoritic materials are shown in figures 2,3 , and 4 , respectively. The enclosed portions of the diagrams are those regions in which asteroids are found. The spectral data were obtained from freshly prepared materials using a Carey 14 spectrophotometer with an attachment to measure nonspecular radiation diffusely scattered at a phase angle of about $60^{\circ}$. The $U-B$ and $B-V$ indexes were calculated from the ratios of reflectivities relative to $\mathrm{MgO}$ smoke at wavelengths of 360,440 , and $550 \mathrm{~nm}$ and thus they correspond to data taken through narrowband filters.

Metallic iron, the $\mathrm{Ni} / \mathrm{Fe}$ meteorites, magnetite, and ilmenite are highly absorbing at all wavelengths and thus have $U B V$ difference indexes that are very close to zero and that may even be slightly negative. Magnetite is the major opaque mineral in terrestrial igneous rocks and ilmenite in lunar materials.

Several nonopaque, rock-forming minerals, such as pyroxene and olivine, have an absorption edge in the near-UV or visible region; and it is this edge that is partly characterized by the $U B V$ indexes. The nature of this edge is uncertain, but it is known to be strongly affected by the presence of Fe (Shankland, 1968). The edge may involve charge transfer, the tail of the $\mathrm{Fe}^{3+}$ UV band, or both.

An extremely important property of this edge is that for most substances it causes the slope of the spectral reflectivity to depend strongly on the size of the particles that make up the reflecting surface. The smaller the size, the 


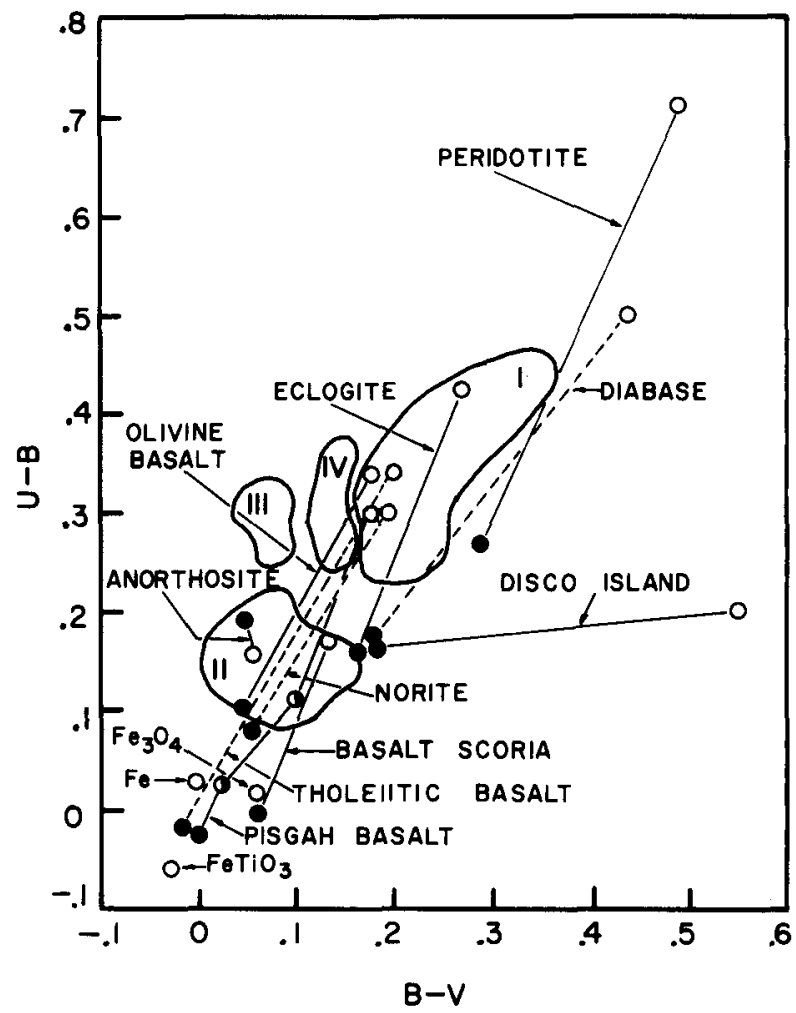

Figure 2.-Color difference diagram for terrestrial rocks. Filled circles indicate solid surfaces; open circles, powder finer than $37 \mu \mathrm{m}$ in grain size; half-filled circles, powders coarser than $37 \mu \mathrm{m}$.

greater the slope and the larger the $U B V$ difference indexes. The reason for this behavior is that a change of particle size has the opposite effect on the reflectivities of opaque and nonopaque materials. As the particle size of an opaque substance is reduced, the surface becomes more complex and a ray requires more reflections, on the average, to escape from the surface. For a weakly absorbing material, as the particle size is decreased, the density of boundary surfaces, which are primarily responsible for scattering the rays out of the medium, increases; thus the average pathlength through the material is decreased, causing reduced absorption.

Hence, the effect of decreasing particle size is to reduce the reflectivity on the short-wavelength side of the edge, where surface scattering dominates, and to increase reflectivity longwards of the edge, where most rays are volume scattered, resulting in an increased slope at the edge. This effect is shown by all the materials in figures 2 to 4 , where filled symbols represent solid, but unpolished, surfaces and open symbols represent powders. The sole exception is anorthosite, in which the edge is well below $360 \mathrm{~nm}$. 


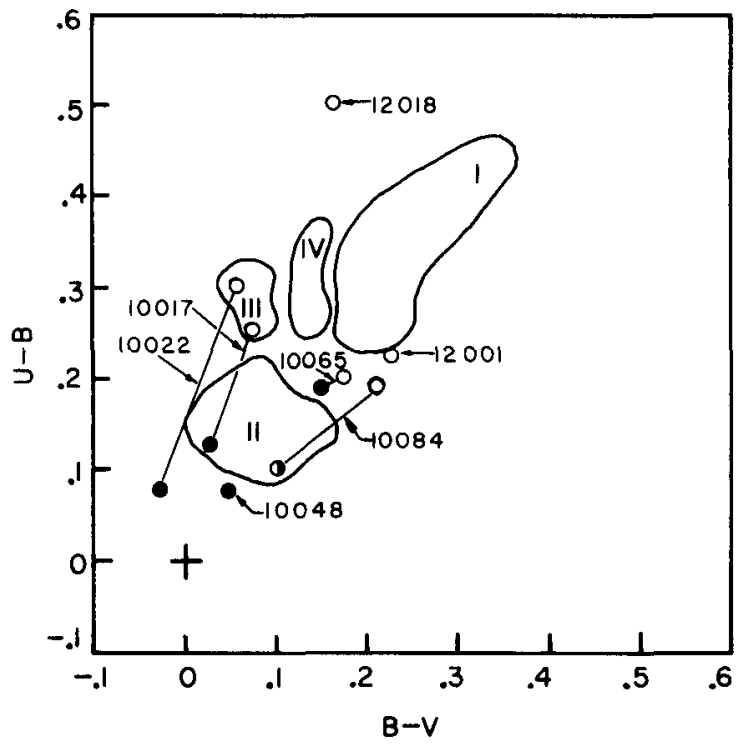

Figure 3.-Color difference diagram for lunar materials. Numbers are NASA designations. Filled circles indicate solid surfaces; open circles, powders finer than $37 \mu \mathrm{m}$ in grain size; half-filled circles, powders coarser than $37 \mu \mathrm{m}$. Nos. 10017, 10022, and 12018 are crystalline rocks; 10048 and 10065 are breccia; 10084 and 12001 are soil.

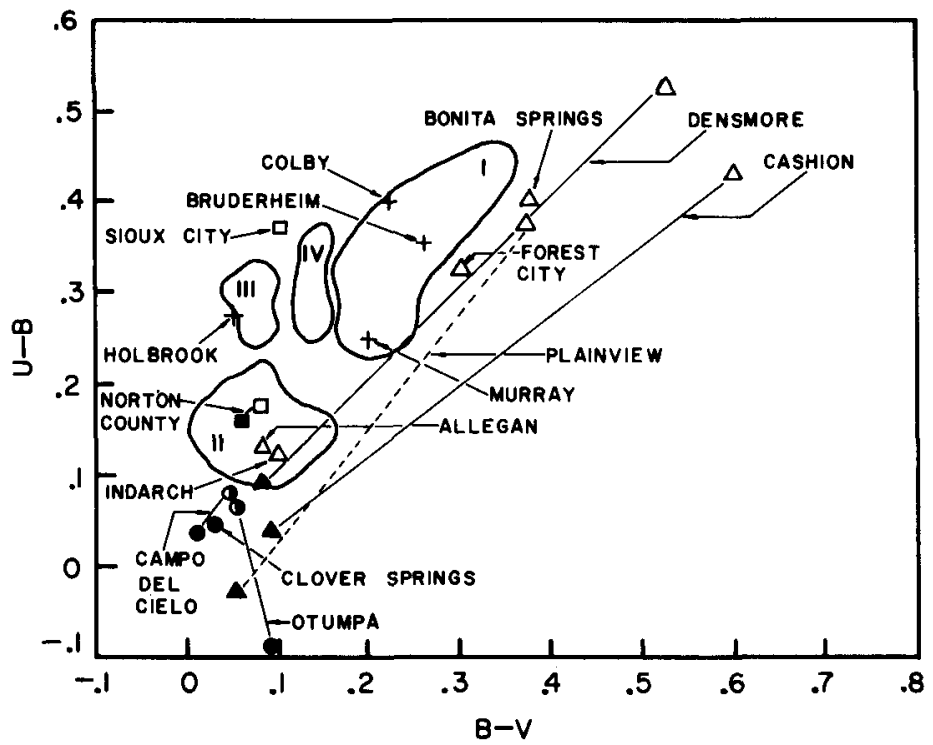

Figure 4.-Color difference diagram for meteorites. Filled symbols indicate solid surfaces; open symbols, powders finer than $37 \mu \mathrm{m}$ in grain size; half-filled symbols, powders coarser than $37 \mu \mathrm{m}$. Plus signs indicate low-iron chondrite powder finer than $37 \mu \mathrm{m}$; circles, irons; triangles, high-iron chondrites; squares, achondrites. 


\section{DISCUSSION OF RESULTS}

\section{UBV Comparisons}

In comparing the data for the asteroids (fig. 1) with those for laboratory materials (figs. 2 to 4 ), several points should be kept in mind.

(1) The colors of the asteroids were obtained with comparatively wide band filters, whereas the laboratory data were taken with the equivalent of narrowband filters.

(2) The laboratory spectra were taken at a phase angle of approximately $60^{\circ}$. Most substances, however, exhibit some reddening with phase. Thus, to be directly compared with the asteroid colors, which have generally been observed at small phase angle, the points of figures 2 to 4 should be moved somewhat inward toward the origin.

(3) The meteorite and asteroidal materials may have undergone unknown degrees of modification. Asteroidal surfaces will be affected by impact melting and vaporization, solar-wind spluttering, and other radiation damage. Meteoritic materials, particularly the finds and old falls in figure 4, will be affected by oxygen and water in the terrestrial atmosphere. The effect of such chemical alteration is to increase the color differences of meteorites over their values in space. However, all of these corrections will only serve to enhance the conclusions that will be made here from the $U B V$ data.

The asteroids all have positive $U-B$ and $B-V$ indexes; that is, they are considerably redder than the Sun. They appear to be arranged into at least three and probably four groups, which are arbitrarily labeled in figure 1 . The Moon appears to belong to color group I. Comparison of figures 1 to 4 discloses several additional interesting features.

(1) None of the asteroids lie in the low color-index region occupied by the iron meteorites and other highly absorbing materials, either in solid or powder forms. Thus, none of the asteroids appear to be pure $\mathrm{Ni} / \mathrm{Fe}$. To increase the $U B V$ indexes, some silicate materials must be added to the iron, which would produce a material resembling stony iron meteorites.

(2) Many other solid surfaces have $U B V$ difference indexes that are smaller than for any asteroid group. Several solid surfaces, as well as powders, lie near the group Il position, but no solid surface corresponds to group I, III, or IV. (The only terrestrial rock that was found to have a large color was peridotite. This rock consists almost entirely of olivine, a transparent, iron-rich mineral. However, no meteorite with a composition similar to pure peridotite is known.) Thus, depending on composition, group II asteroids could have solid or powdered surfaces or mixtures, whereas asteroids of the other 
groups appear to have surfaces that are morphologically similar to powdered silicate rocks and stony meteorites.

(3) The direction of change of the color difference indexes upon pulverization lies parallel to the displacements of groups I, III, and IV from group II, and also depends on content of metallic iron. Powders of high-iron chondritic meteorites and Disco Island basalt, which contain metallic iron, tend to lie toward the upper right part of the diagrams. Powders of most of the terrestrial and lunar basalts, achondrites, and low-iron chondrites tend to be displaced farther toward the upper left. Many of the high-iron chondrites lie outside the asteroid fields, possibly because of oxidation by the terrestrial environment.

(4) Lunar soil has different $U B V$ values than pulverized lunar crystalline rocks, even though the two materials have similar compositions. Hapke, Cassidy, and Wells (1971) have suggested that this difference is due to a component in the lunar soil that has been deposited directly from a vapor. The vapor is probably generated by meteorite impacts and by solar-wind sputtering. These processes, however, may be expected to be less important in the asteroid belt than on the Moon because of the lower relative velocity of meteorite collisions and the reduced flux of solar wind.

(5) The correlations of $U B V$ position with metallic iron content suggests that it eventually may be possible to associate the parents of various types of meteorites with different asteroid groups, although to attempt specific identifications before the effects of the space and terrestrial environments are understood would be premature. The various asteroid groups also may represent different conditions of surface pulverization.

\section{OTHER OPTICAL COMPARISONS}

Other optical properties of asteroids and the Moon are summarized in table I. The albedos of most of the asteroids are sufficiently low that their surfaces could consist of either solids or powders of iron-rich material. However, Vesta has such a high albedo that its surface can only be a pulverized, iron-poor substance, such as low-iron achondrite. It is well known that the strongly backscattering nature of the lunar photometric function, represented in table I by $\partial m_{v} / \partial \alpha$, is due to blocking and shadowing of sunlight in the fine-grained, dark particles of lunar soil (Hapke and Van Horn, 1963). The asteroids of table I have phase function slopes that are as large or larger than the Moon's, implying surfaces of equal or greater complexity, which could be readily produced by powder.

The opposition effect is not as well understood as the backscatter effect, but it is known to be much more pronounced for fine powders than for coarse surfaces. Table II gives the opposition effect for some olivine basalt powders as 
TABLE II.-Opposition Effect of Basalt Powders

\begin{tabular}{l|r|r|r|r}
\hline \multirow{2}{*}{$\begin{array}{c}\text { Size, } \\
\mu \mathrm{m}\end{array}$} & \multicolumn{2}{|c|}{ Opposition effect } & \multicolumn{2}{c}{ Geometric albedo, percent } \\
\cline { 2 - 5 } & $\mathrm{N}$ & $\mathrm{I}$ & $\mathrm{N}$ & $\mathrm{I}$ \\
\hline 10 & 1.20 & 1.28 & 45 & 11 \\
50 & 1.04 & 1.11 & 14 & 10 \\
Solid & - & 1.12 & 8 & 11 \\
\hline
\end{tabular}

$\mathrm{I}=$ irradiated by a simulated solar wind; $\mathrm{N}=$ not irradiated.

a function of particle size. Only particles smaller than about $50 \mu \mathrm{m}$ seem to have an opposition effect as large as that of the asteroids.

The negative branches of the polarization-phase curves of asteroids seem to be generally similar to the Moon's, although the details vary from body to body. As shown by Lyot (1929), a lunar-type negative branch requires a fine-grained, dark powder. The negative branch can be altered by changing either the grain size or the opacity of the particles. The polarization curve for Ceres seems to correspond better to coarse-grained material than to fine powder, although Ceres exhibits strong backscatter.

In conclusion, the optical properties of asteroids are generally consistent with a surface coating of fine, loose powder, similar to the dust that covers the surface of the Moon. Similar conclusions were reached earlier by Gehrels, Coffeen, and Owings (1964). It is possible, of course, to imagine other materials with a morphology that is fine scaled and complex, such as some sort of volcanic or impact-generated foam or scoria. However, dust appears to be a ubiquitous material in the solar system, and there appear to be no strong arguments against the presence of dust on the surfaces of most of the asteroids. The dust is generated readily by collisions between asteroids of all sizes. It will cling readily to surfaces of asteroids, in spite of their low gravity, by the same electrostatic and intermolecular van der Waals and covalent adhesive forces that cause grains of lunar soil to clump together. This pulverized layer needs to be only a few layers thick to account for the observed optical properties; however, there is no reason to suppose that it is not much thicker in places, particularly in concavities where it is protected from disturbance.

\section{ACKNOWLEDGMENT}

I thank E. Skopinski for his valuable assistance in the preparation of this paper. This research was supported by NASA grant NGR 39-011-085.

\section{REFERENCES}

Allen, C. 1963, Astrophysical Quantities. Athlone Press. London.

Burns, R. 1970, Mineralogical Applications of Crystal Field Theory. Cambridge Univ. Press. London.

Dollfus, A. 1961, Polarization Studies of Planets. Planets and Satellites (eds., G. Kuiper and B. Middlehurst), p. 343. Univ. of Chicago Press, Chicago. 
Garbuny, M. 1965, Optical Physics. Academic Press, Inc. New York.

Gehrels, T. 1956, Photometric Studies of Asteroids. V. The Lightcurve and Phase Function of 20 Massalia. Astrophys. J. 123, 331.

Gehrels, T. 1970, Photometry of Asteroids. Surfaces and Interiors of Planets and Satellites (ed., A. Dollfus), p. 319. Academic Press, Inc. New York.

Gehrels, T., Coffeen, D., and Owings, D. 1964, Wavelength Dependence of Polarization. III. The Lunar Surface. Astron. J. 69, 826.

Goldstein, R. 1968, Radar Observations of Icarus. Science 162, 903.

Hapke, B., Cassidy, W., and Wells, E. 1971, The Albedo of the Moon: Evidence for Vapor-Phase Deposition Processes on the Lunar Surface. Proc. Apollo 12 Lunar Sci. Conf. Geochim. Cosmochim. Acta, to be published.

Hapke, B., and Van Horn, H. 1963, Photometric Studies of Complex Surfaces, With Applications to the Moon. J. Geophys. Res. 68, 4545.

Harris, D. 1961, Photometry and Colorimetry of Planets and Satellites. Planets and Satellites (eds., G. Kuiper and B. Middlehurst), p. 272. Univ. of Chicago Press. Chicago.

Lyot, B. 1929, Recherches sur la Polarisation de la Lumière des Planètes et de Quelques Substances Terrestres. Ann. Observ. Paris Meudon 8(1).

McCord, T., Adams, J., and Johnson, T. 1970, Asteroid Vesta: Spectral Reflectivity and Compositional Implications. Science 168, 1445.

McCord, T., and Johnson, T. 1970, Lunar Spectral Reflectivity (0.30 to 2.50 Microns) and Implications for Remote Mineralogical Analysis. Science 169, 855.

Miner, E., and Young, J. 1969, Photometric Determination of the Rotation Period of 1566 Icarus. Icarus 10, 436.

Shankland, T. 1968, Band Gap of Forsterite. Science 161, 51 .

\section{DISCUSSION}

BRECHER: The spectral reflectivity data of McCord, Adams, and Johnson (1970) indicated the presence on Vesta's surface of material similar to basaltic achondrites. But these are known to be similar to the silicate part of the mesosiderites while a large ( $<50$ percent by volume) $\mathrm{Ni} / \mathrm{Fe}$ content would be more consistent with Schubart's density for Vesta. Could your data rule out the presence of $\mathrm{Ni} / \mathrm{Fe}$ in Vesta's surface material?

HAPKE: No. The spectrum of metallic $\mathrm{Ni} / \mathrm{Fe}$ is essentially flat; therefore, its presence will reduce the contrast of silicate spectral bands but will not completely obscure them.

CHAPMAN: It is true that the mineralogical composition of the silicate portion of some mesosiderites should yield a spectral reflectivity similar to that our group has observed for Vesta. Because the albedo of the metallic portion of such meteorites is so much lower than the albedo of the silicate portion, it would make little change in the shape of the reflectivity curve (except possibly in the UV), even if present in equal proportions. The presence of such a dark component, with a relatively featureless spectral reflectivity, might help to explain why the albedo of Vesta, implied by some recent diameter estimates, is lower than might be expected for the identified silicates.

\section{DISCUSSION REFERENCE}

MoCord, T., Adams, J., and Johnson, T. 1970, Asteroid Vesta: Spectral Reflectivity and Compositional Implications. Science 168, 1445. 